\title{
Preparing to Stop Action Increases Beta Band Power in Contralateral Sensorimotor Cortex
}

\author{
Vignesh Muralidharan ${ }^{1}, X_{\text {Xinze }} \mathbf{Y u}^{1}$, Mike X Cohen $^{2}$, and Adam R. Aron ${ }^{1}$
}

\begin{abstract}
How do we prepare to stop ourselves in the future? Here, we used scalp EEG to test the hypothesis that people prepare to stop by putting parts of their motor system (specifically, here, sensorimotor cortex) into a suppressed state ahead of time. On each trial, participants were cued to prepare to stop one hand and then initiated a bimanual movement. On a minority of trials, participants were instructed to stop the cued hand while continuing quickly with the other. We used a guided multivariate source separation method to examine oscillatory power changes in presumed sensorimotor cortical areas. We observed that, when people prepare to stop a hand, there were above-baseline beta band power increases (12-24 Hz) in contralateral cortex up to a second earlier. This increase in beta band
\end{abstract}

\section{INTRODUCTION}

One aspect of self-control is preparing to stop. A better understanding of how people prepare to stop is important for the basic science of cognitive control, for everyday life (one is often in situations where one is provoked by stimuli that afford response tendencies), and also for clinical disorders such as impulse control deficits and Tourette's syndrome.

The situation of the preparing-to-stop state has been modeled in the laboratory with variants of a task called proactive selective stopping (Raud \& Huster, 2017; Cowie, MacDonald, Cirillo, \& Byblow, 2016; Xu, Westrick, \& Ivry, 2015; Lavallee, Meemken, Herrmann, \& Huster, 2014; Smittenaar, Guitart-Masip, Lutti, \& Dolan, 2013; Cai, Oldenkamp, \& Aron, 2011; Claffey, Sheldon, Stinear, Verbruggen, \& Aron, 2010; Aron \& Verbruggen, 2008; Coxon, Stinear, \& Byblow, 2007; for a review, see Duque, Greenhouse, Labruna, \& Ivry, 2017). In such tasks, on each trial, the participant is given a cue about which possible responses they might have to stop a few seconds later. On most trials, they proceed to make two responses, but sometimes they have to stop one while continuing quickly with the other.

${ }^{1}$ University of California, San Diego, ${ }^{2}$ Radboud University

(C) 2019 Massachusetts Institute of Technology power in the proactive period was functionally relevant because it predicted, trial by trial, the degree of selectivity with which participants subsequently stopped a response but did not relate to movement per se. Thus, preparing to stop particular response channels corresponds to increased beta power from contralateral (sensorimotor) cortex, and this relates specifically to subsequent stopping. These results provide a high temporal resolution and frequency-specific electrophysiological signature of the preparing-to-stop state that is pertinent to future studies of mitigating provocation, including in clinical disorders. The results also highlight the utility of guided multivariate source separation for revealing the cortical dynamics underlying both movement and response suppression.

The speed of continuation is a measure of the selectivity of stopping, and it has been argued that selective stopping is much aided by encoding the cue at the beginning of the trial and already implementing that cue as a selective suppression over response channels (Majid, Cai, Corey-Bloom, \& Aron, 2013; Cai et al., 2011). Evidence supporting this is that, when one prepares to stop the right hand, there is, in the proactive period, reduced corticospinal excitability of the right hand (measured using single-pulse TMS; Majid et al., 2013; Cai et al., 2011; Claffey et al., 2010).

Here, we set out to test the cortical correlates of the preparing-to-stop state using scalp EEG. This method has many advantages, including measuring both left and right presumed cortical sensorimotor areas at the same time, for different frequency bands and with high temporal resolution to get at subsecond dynamics with continuous changes. Yet there are challenges with scalp EEG, including the fact that the signal-to-noise ratio of individual electrodes is poor, that many electrodes reflect the same underlying signals owing to volume conduction, and that sensorimotor responses may be maximal at different electrodes for different participants (because of cortical folding, motor cortex organization, and electrode placement).

It is possible, however, to derive more specific sources that have higher signal-to-noise ratio by using various 
methods of spatial filtering, such as the Laplacian (e.g., Pfurtscheller \& Solis-Escalante, 2009) and independent component analysis (Makeig, Bell, Jung, \& Sejnowski, 1996). Here, we used a method known as guided multivariate source separation and specifically one that used generalized eigenvalue decomposition (GED; for earlier uses in scalp EEG, see Cohen, 2017; Blankertz, Tomioka, Lemm, Kawanabe, \& Müller, 2008; Parra \& Sajda, 2004). This approach is "guided" because one starts with a prior about the topography of the spatial filter (in our case that it would be contralateral to the hand that one is preparing to stop), a prior about the frequency band of interest (in our case we focused on the beta band, $12-24 \mathrm{~Hz}$, given a substantial literature relating sensorimotor beta to an akinetic state; Kilavik, Zaepffel, Brovelli, MacKay, \& Riehle, 2013; Engel \& Fries, 2010), and a prior about the time of interest (we focused on a time period of $1 \mathrm{sec}$ after the cue to prepare to stop; Cai et al., 2011). With this method, we aimed to derive the weights or spatial filters over the right sensorimotor cortex that optimally separates prepare-to-stop-left trials from prepare-to-stop-right trials, and vice versa, for the left sensorimotor cortex.

We used a bimanual task to get at selective suppression; that is, how participants stopped one hand and continued with the other. Whereas selective suppression can also be done within one hand (e.g., Coxon et al., 2007), here we used two hands to get a clean separation of sensorimotor components in EEG.

We had two hypotheses. First, when preparing to stop a hand, there would be increased beta band power in the contralateral sensorimotor area. Second, this increased sensorimotor beta power in the proactive period would relate to the subsequent stopping process, specifically that participants with more proactive sensorimotor beta would, when they had to stop, target their suppression process more specifically to a particular hand.

\section{METHODS}

\section{Participants}

Sixteen right-handed participants ( 4 men, 12 women; mean age $=21.13$ years, range $=18-35$ years) provided written consent in accordance with the institutional review board guidelines of the University of California, San Diego.

\section{Procedure}

Sixty-four channel EEG was recorded from an EasyCap slim electrode system with the actiChamp system (Brain Vision LLC). The task was run in MATLAB (R2014b) using Psychtoolbox 3 (Brainard, 1997). After EEG capping, an impedance check was done with PyCorder. The EEG data were sampled at $1024 \mathrm{~Hz}$ with a $60-\mathrm{Hz}$ notch filter.

The participant placed the left and right little fingers on the keys "Z" and "/" and the left and right index fingers on "V" and "M" on a regular keyboard. Each trial began with one of four cues: Maybe Stop Left (MSL), Maybe Stop Right (MSR), Go Left (GL), or Go Right (GR). These occurred with equal likelihood and were presented in the center of a black screen for 500 msec. After the cue,there was a 1-sec interval, and then four circles were presented in a horizontal row, corresponding to the four fingers. The display of these circles depended on whether it was an MSL/MSR or GL/GR trial.

For MSL/MSR, either two inner or two outer circles were filled in blue (go signal) and the other two were filled with white (Figure 1). The participant needed to make simultaneous bimanual response to the two blue circles using the corresponding fingers as fast as possible: with two index fingers for the inner circles and two little fingers for the outer circles. These trials could be go trials

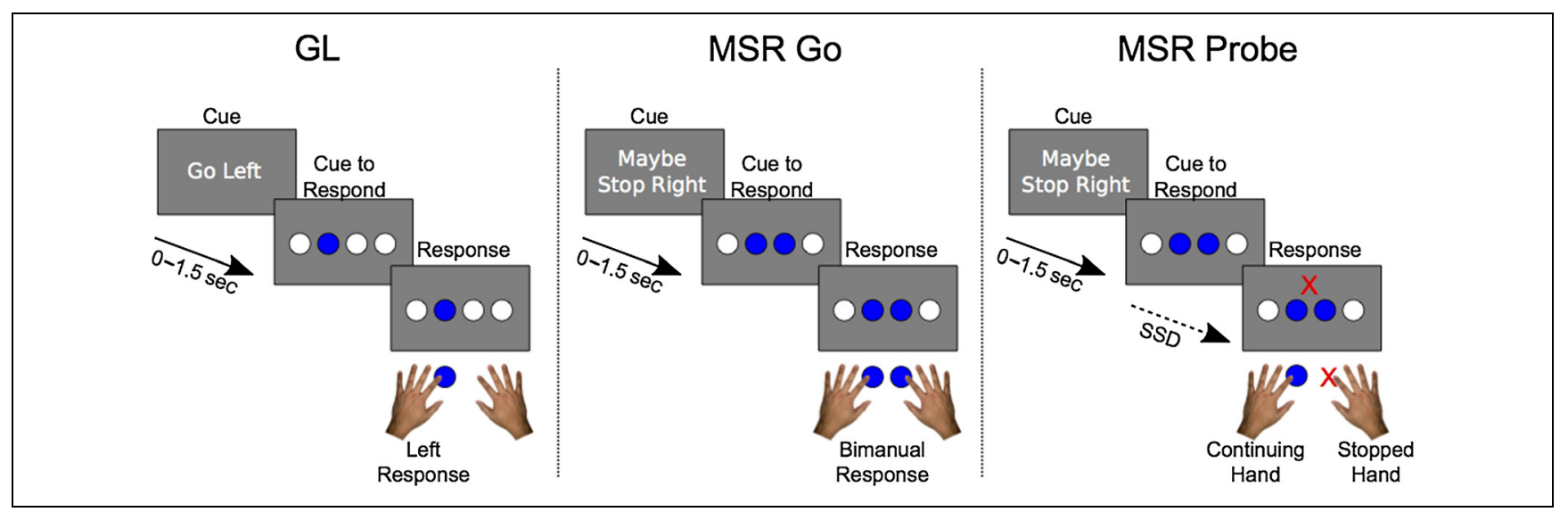

Figure 1. The task. Left: A GL trial where participants prepare to move their left hand. The four circles correspond to the index and little fingers of each hand. In this case, the actual response finger could be either index or little finger of the left hand. Middle: An MSR go trial where the participants get ready to stop their right hand while later needing to make a bimanual response (no stop signal or "probe"). Right: An MSR probe trial where again the participants prepare to stop the right hand, initiate a bimanual movement, and then, when the stop signal (red " $\mathrm{X}$ ") occurs, they stop their right hand and quickly continue with their left. 
(67\%) or probe trials (33\%; requiring the participant to try to stop). On go trials, no stop signal occurred, and the participant made a fast bimanual response. The word "decoupled" was presented after the response if the RT difference between the two fingers was larger than $70 \mathrm{msec}$ (this was rare, $2.17 \%$ of the trials). On probe trials, a red cross (stop signal) was presented after the go signal and in the middle of the screen (between the two inner circles), which was uninformative about which response to stop. This "forced" participants to encode and use the initial cue to prepare to stop. When the stop signal occurred, the participant tried to stop one hand while continuing with the other as fast as possible. The program dynamically adjusted the delay between the go signal and the stop signal (the stop signal delay [SSD]) based on the participant's performance on stop trials. If the participant successfully stopped the cued hand in the current probe trial, the SSD increased by $50 \mathrm{msec}$ for the next probe trial; if the participant failed, the SSD decreased by $50 \mathrm{msec}$ for the next probe trial.

For the GL/GR conditions, only one of the four circles was filled in blue (go signal). If the cue was GL, one of the left two circles was blue, and if GR, then one of the right two circles was filled blue. The participant needed to respond to the blue circle using the corresponding finger (index or little). These conditions prepared the participant to respond with a particular hand with no possibility of stop signals.

There were six blocks of 80 trials in total. Each block contained 20 trials of MSL, MSR, GL, and GR in a pseudorandom order with no more than two consecutive probe trials. EEG markers were sent at four time points: the onset of the cue, the onset of the go signal, the onset of the stop signal, and the response. Here, we focus only on the onset of the cue.

\section{Behavioral Analysis}

Following Cai et al. (2011), we calculated go accuracy for the MSL and MSR conditions (i.e., the proportion of trials on which the participant pressed the correct buttons: inner or outer) and on GL and GR conditions (i.e., the correct button), probe accuracy (i.e., the proportion of trials in which the participant correctly stopped in MSL and MSR conditions), probe alternative RT (i.e., the average RT of the continuing hand for the probe trials in the MSL and MSR conditions), the SSD (i.e., the delay between the onset of the go signal and the stop signal), the stop signal RT (go signal RT minus SSD), and the so-called "stopping interference effect." Some earlier articles calculated this interference effect as the probe alternative RT minus the go RT of the same hand (i.e., for MSL, the left hand stops and the right hand continues, so the stopping interference effect is probe alternative RT for the right hand minus RT for the right hand on go trials; Aron \& Verbruggen, 2008). However, this method overestimates the stopping interference effect (see also
Xu et al., 2015). Here we calculated the stopping interference effect as the probe alternative RT minus the go RT when only taking the right hand side of the go distribution (i.e., taking the mean of the right "half," i.e., the slower go trials). In more detail, the rationale for using the right hand side of the go distribution is as follows. One wants to compute how much slower the continuing hand is when one stops the other, compared with the continuing hand when no stopping was required. Yet trials that are successfully stopped have slower go processes than the average of all trials (Logan \& Cowan, 1984); by taking the right hand side (slower) of the distribution on go trials, one has a better baseline for comparison.

\section{EEG Analysis}

The EEG data were down-sampled from 1024 to $512 \mathrm{~Hz}$, band-passed filtered from 2 to $100 \mathrm{~Hz}$, rereferenced to the mastoids, and manually inspected to remove bad channels and noisy stretches of data. Following this, the data were rereferenced to the average. The initial referencing to the mastoids was done to identify noisy electrodes and remove them from the analysis. The rereferencing to the mean was then done as a way to reduce hemisphere bias.

Whereas picking a single electrode (or set of electrodes) may not cleanly separate left versus right responses because of volume conduction and individual anatomical/functional variability, we resorted, as explained above, to a guided source separation method known as GED (for earlier uses in scalp EEG, see Cohen, 2017; Blankertz et al., 2008; Parra \& Sajda, 2004). For each participant for MSL and MSR, our analysis steps were as follows:

1. Epoching the EEG data into 1.5 -sec trials ( -0.5 before the cue to 1 sec after it; Figure 2A).

2. Narrow band filtering the data at each frequency ranging from 12 to $24 \mathrm{~Hz}$ using a frequency domain Gaussian kernel with a FWHM of $5 \mathrm{~Hz}$. (The choice of using a window of 12-24 Hz was motivated by the a priori hypothesis of the beta band being implicated in a suppressive state in sensorimotor cortex [see Introduction]. We ended a little lower [24 Hz] than the typical $30 \mathrm{~Hz}$ because it required manually reviewing fewer components across all frequencies and because initial inspection showed participants had components below the $24-\mathrm{Hz}$ range).

3. Computing covariance matrices at every frequency for both sets of trials $\left(C_{\mathrm{MSL}}\right.$ and $\left.C_{\mathrm{MSR}}\right)$ for the time period of 0-1000 msec (i.e., from the onset of the cue for $1000 \mathrm{msec}$ ) and running GED (Figure 2B).

4. Selecting the best component for each participant (see below).

We now describe Step 3 in more detail. For the MSR condition, the GED equation was

$$
C_{\mathrm{MSL}}^{-1} C_{\mathrm{MSR}} W_{\mathrm{D}}=W_{\mathrm{D}} \Lambda
$$


Figure 2. GED applied to the EEG data from the task. (A) The time window for GED was from 0 to $1000 \mathrm{msec}$ after the onset of the cue (here, a MSR cue). (B) The representation of the GED implementation in one participant: Here, GED is performed for MSR versus MSL trials and $C_{\mathrm{MSL}}$ and $C_{\mathrm{MSR}}$ are the covariance matrices (for 0-1000 msec) for the MSL and MSR conditions, respectively. $W_{\mathrm{D}}$ is the eigenvector corresponding to the eigenvalue $(\Lambda)$.

(C) The eigenvalue spectrum was obtained from the same participant (in B), and the embedded topography is the filter forward model (activation $=C_{\mathrm{MSR}} W_{\mathrm{D}}$ ).

The forward model corresponding to the top eigenvalue has a left sensorimotor topography. (D) The power ratio between the mean power in MSR and MSL in the $0-1000 \mathrm{msec}$ window. The threshold is 1 for selection of the spatial filter.

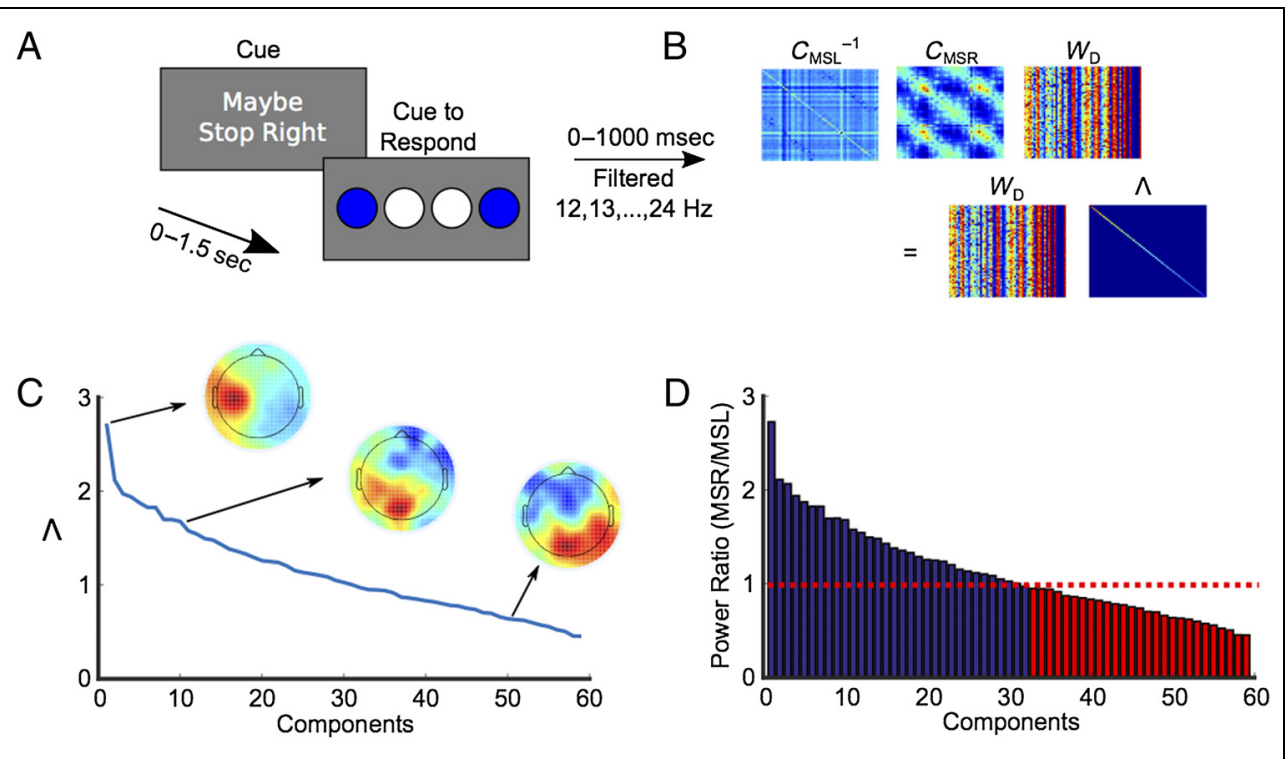

This provides the weights or spatial filters, $W_{\mathrm{D}}$, which optimally separate MSR trials from MSL. The eigenvalues $\Lambda$ provide the variance explained by the spatial filter. Projecting the weights onto the covariance matrix produces the activation map, which can then be visualized as the topographical map for physiological interpretation (Haufe et al., 2014). The topography of the filter, also known as the filter forward model, was estimated as $t_{\text {map }}=C_{\mathrm{MSR}} W_{\mathrm{D}}{ }^{n}$, where $W_{\mathrm{D}}^{n}$ is the spatial filter belonging to the $n$th eigenvalue.

For the MSL condition, the GED equation was

$$
C_{\mathrm{MSR}}^{-1} C_{\mathrm{MSL}} W_{\mathrm{D}}=W_{\mathrm{D}} \Lambda
$$

We now describe Step 4, the selection of the best components, in more detail. For a participant, in the MSL or MSR condition, we proceeded as follows. First, we restricted the selection of components to the beta frequency range (i.e., $12-24 \mathrm{~Hz}$ ). Second, a particular spatial filter was then selected based on three criteria: (a) the amount of variance explained by the component (we restricted our component selection to the top 6 eigenvectors), (b) the spatial activation pattern (topography) of the component (when comparing MSR vs. MSL, we looked for a source that was left lateralized and over centrofrontal/central/centroparietal electrodes; an illustration of the eigenvalue distribution and the topography of each eigenvector corresponding to that eigenvalue is shown in Figure 2C), and (c) the power ratio (i.e., the ratio of mean power in the $0-1000 \mathrm{msec}$ period in both the conditions in the GED). To do this, the power time series for $0-1000$ msec was obtained by projecting the bandpass filtered data onto the specific spatial filter. For example, when performing GED for MSR versus MSL, the power ratio was $P_{\mathrm{MSR}} / P_{\mathrm{MSL}}$ and the threshold for selection was 1 , and the particular spatial filter was considered for selection if the power ratio was greater than 1 (Figure 2D).

\section{Frequency-Specific Power Extraction and Hypothesis Testing}

Our first hypothesis was that preparing to stop would elicit increased beta band power over the response hand contralateral cortex. Note that our method of using GED does not guarantee this result-there could be some difference in the beta band between MSL and MSR conditions where the underlying data reflect a beneathbaseline reduction in both conditions. To test the hypothesis, for each participant, we projected the temporally filtered data onto the spatial filter (e.g., the MSR and MSL trials onto the left sensorimotor (SM) spatial filter and the MSL and MSR trials onto the right SM spatial filter) and then estimated the power time course. We did this as follows. First, the data were filtered at the frequency where we obtained the spatial filter for a particular participant (e.g., for Participant P1, the left spatial filter was derived for $12 \mathrm{~Hz}$ and the right spatial filter was derived for $15 \mathrm{~Hz}$ ) and then projected onto the corresponding spatial filter. Fast Fourier analysis was done using the Hilbert transform. The power time course was then computed by squaring the magnitude of the analytic envelope. The result was then decibel-normalized using a pretrial baseline power of -500 to $-200 \mathrm{msec}$. The resulting baselinenormalized power estimate was then plotted across the 1-sec time period after the cue for each participant for each of MSL and MSR conditions for each of the left 
and right sensorimotor filters. For this analysis, timelocked to the MSL and MSR cues there were an average of 67 and 66 trials after artifact rejection.

Our second hypothesis was that the increased beta power in contralateral cortex would relate to trial-by-trial variability in subsequent stopping. Although we did not have a single-trial estimate of stopping, we had a proxy - the speed of the continuing hand. For example, if the cue instructed MSR and the participant stopped the right hand selectively, then the time to continue the left hand would be short. Trial by trial variability in the continuing hand thus could possibly serve as an index of a selective stopping process. We now asked, how does the RT of the continuing hand relate to the earlier beta power? To do this, we calculated the mean power (extracted as above) for $0-1000 \mathrm{msec}$ in the cue period for each trial. For each participant, we then correlated these values against the probe alternative RT. This produces an $r$ value, which was tested against 0 at the group level. For this analysis, time-locked to the MSL and MSR cues, but only on successful stop (probe) trials, there were an average of 11 and 12 trials after artifact rejection.

\section{Cross-validation}

Because GED involves designing a spatial filter that optimally separates two covariance matrices, it is prone to overfitting - that is, the approach may work well on the data used to fit the model but then fail to generalize to unseen data. The brain/behavior analysis described above is immune to this concern, because the beta band power correlation with behavior was not used to derive the filters. But results from the first analysis (above-baseline power increase) could reflect overfitting. Thus, we redid the component selection step using a leave in/out crossvalidation procedure. For each participant, the trials were split into a training set (90\%; used to design the spatial filter) and a testing set (10\%; used to apply the filter to). Ten such validation blocks were set up to make sure that every trial had a chance to be in the training and testing sets. GED was performed on the $90 \%$ data set for every block in the same manner as mentioned in the above section (see Steps 1-3). In each block of 10 , the component with the largest spatial correlation to the original filter map was then selected (this correlation was simply a pairwise correlation between the map values of each electrode in each spatial filter). Then, the power time course was extracted by projecting the test data (10\% trials) onto this filter. The average time course over the 10 blocks was then computed.

\section{RESULTS $^{1}$}

\section{Behavior}

Performance was similar to earlier studies with the same task (Majid et al., 2013; Cai et al., 2011; Claffey et al., 2010; Table 1). Accuracy on go trials was high, and the difference in RT between the two hands was not significantshowing that participants did not uncouple the hands on go trials. The probability of stopping (probe accuracy) was about $58 \%$, not far off the approximate $50 \%$ that is often converged upon by the staircase. Most importantly, the selectivity of stopping (the stopping interference effect) was a mere $81 \mathrm{msec}$ on average. This suggests that

Table 1. Group Mean Behavioral Data

\begin{tabular}{|c|c|c|c|c|}
\hline & $M S L$ & MSR & $G L$ & $G R$ \\
\hline Go cue RT (sec) & $0.560(0.087)$ & $0.556(0.088)$ & $0.434(0.050)$ & $0.425(0.044)$ \\
\hline Go alternative RT (sec) & $0.562(0.088)$ & $0.556(0.091)$ & - & - \\
\hline Go alternative slow RT (sec) & $0.683(0.123)$ & $0.660(0.125)$ & - & - \\
\hline Probe alternative RT (sec) & $0.746(0.139)$ & $0.758(0.150)$ & - & - \\
\hline Stopping interference (sec) & $0.063(0.067)$ & $0.098(0.074)$ & - & - \\
\hline Go accuracy (\%) & $92.49(3.50)$ & $93.38(5.01)$ & $98.75(1.14)$ & $99.06(1.13)$ \\
\hline Probe accuracy (\%) & $57.29(8.17)$ & $58.51(5.60)$ & - & - \\
\hline $\mathrm{SSD}(\mathrm{sec})$ & $0.252(0.067)$ & $0.257(0.068)$ & - & - \\
\hline Stop signal RT (sec) & $0.308(0.039)$ & $0.301(0.040)$ & - & - \\
\hline
\end{tabular}

Values are provided for MSL trials, MSR trials, GL trials, and GR trials. Go cue RT reflects the mean RT (and standard deviation) of go trials (no stop signal) for the hand that definitely will respond, for example, on MSL trials, it is the right hand. Go alternative RT is mean RT of go trials for the hand that might have to stop (but on go trials where no stop signal occurred). Go alternative RT slow is similar except now only the slow half of the distribution is taken. Probe alternative RT is the mean RT of the continuing (nonstopped hand) on trials where the participant correctly stopped. The stopping interference effect is the probe alternative RT minus the go alternative slow RT: This is a key variable that indicates the selectivity of stopping (i.e., the value would be zero if stopping were perfectly selective-it would not interfere with the continuing hand). Go accuracy is whether participants pressed the correct buttons on go trials (e.g., with both index fingers or both pinkie fingers). Probe accuracy is the probability of correctly stopping the cued hand when a stop signal occurred (e.g., on an MSL trials, the left hand is stopped and the participant continues with the right hand). The SSD is the delay between the go signal and the stop signal. SSRT is the stop signal RT, that is, mean go cue RT minus SSD. 


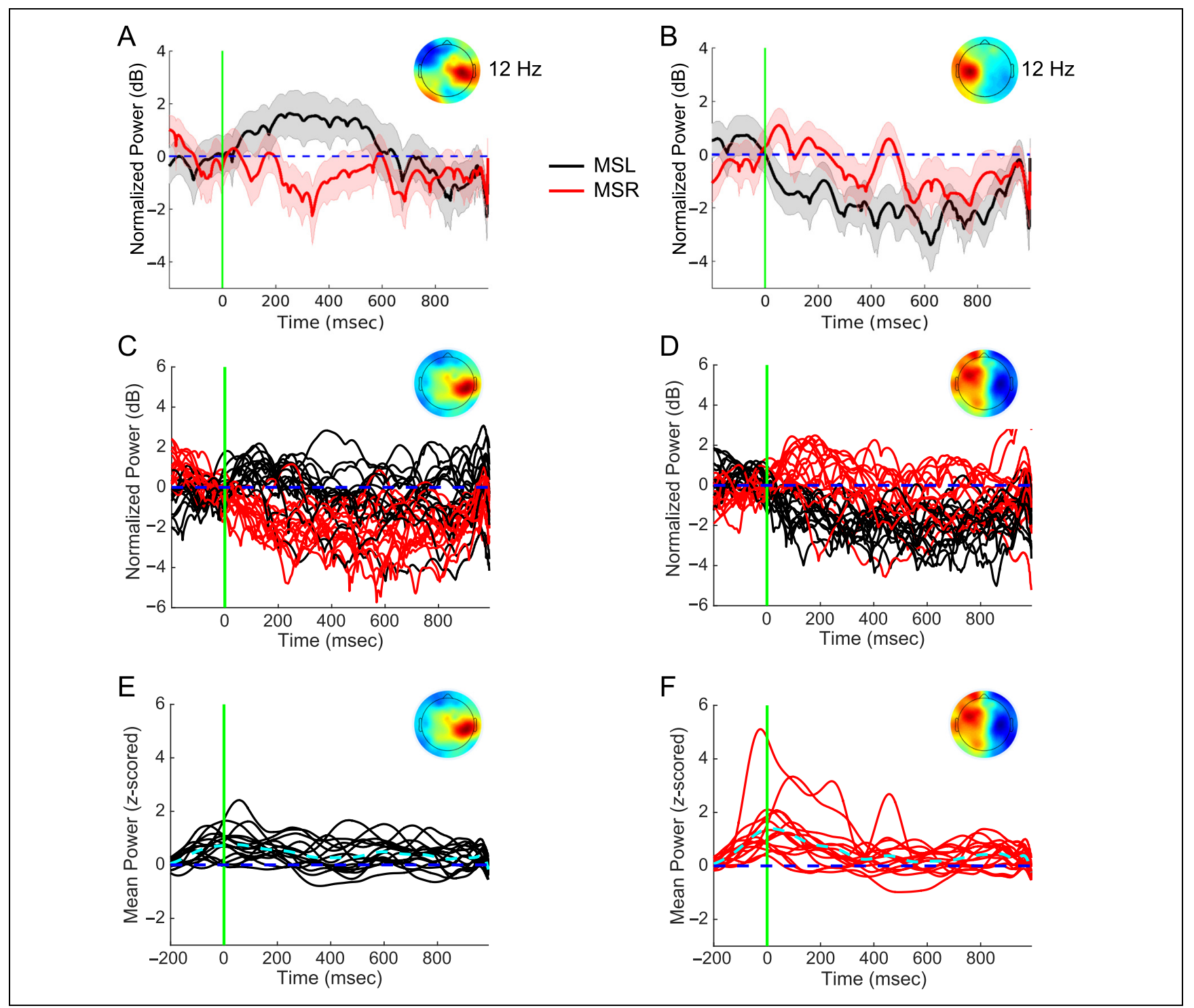

Figure 3. Preparing to stop a hand is associated with increased beta band power in contralateral sensorimotor areas. (A) A single participant example of a right sensorimotor filter. Mean power at $12 \mathrm{~Hz}$ for 0-1000 msec from that filter is shown for MSR and MSL (the shaded area represents the SEM across trials). (B) The same is shown for the left sensorimotor filter. C and D show the mean power for all the participants for the right and the left sensorimotor filters, respectively. E and F are the leave in/out cross-validated power for the MSL and MSR trials for the right and left sensorimotor filters, respectively.

participants were encoding the cue, and we would argue based on prior studies (Claffey et al., 2010), using it ahead of time to proactively suppress response tendencies (by contrast, global stopping should cancel everything and involve a restart, which would makes this value much longer Xu et al., 2015; Coxon et al., 2007). Thus, the behavioral results show that participants performed the task very well and, on average, were probably stopping selectively, rather than doing a global stop and restart.

\section{Preparing to Stop Is Associated with Increased Beta Band Power in Contralateral Cortex}

Our first hypothesis was that preparing to stop would lead to increased beta band power in the contralateral cortex. To test this, we extracted power time series for the left and right SM spatial filters for each participant separately (right spatial filter, derived by performing MSL vs. MSR using GED, and left spatial filter, derived by performing MSR vs. MSL using GED). Figure 3 (A, B) shows the power time course for a representative participant for the right and left spatial filters. Figure 3 (C, D) shows this for all participants. These data show that when the cue is MSL there is increased beta power over right SM and when the cue is MSR there is increased beta power over left SM.

However, as these results arose from data that were also used to derive the filters in the first place, we performed validation with the leave in/out cross-validation procedure. In this case, we used some of the data to 
generate the filters ("training set") and the left-out data for plotting ("testing set"). The pattern of results was the same (increased beta power in contralateral cortex in the preparing-to-stop period), indicating that the result was not biased by overfitting (Figure 3E, F).

\section{Beta Power When Preparing to Stop Is Related to the Behavioral Selectivity of Stopping}

Our second hypothesis was that the increased beta power in the proactive period would be functionally relevant for subsequent stopping. To test this, we extracted mean beta power in the proactive period for each participant and each trial in each MSL and MSR condition. Across successful probe (stop) trials, we then correlated this beta power against the RT of the continuing (nonstopped) hand to generate an $r$ value in each participant. Then, at the group level, for MSL and MSR separately, we tested if these $r$ values were different from zero. Indeed, across participants, there was a significant negative correlation between the mean beta power and the probe alternative RT $(t=2.6798, p=.01, d=1.38$ for MSR trials; $t=2.6781, p=.01, d=1.38$ for MSL trials): that is, on successful stop trials where earlier beta power

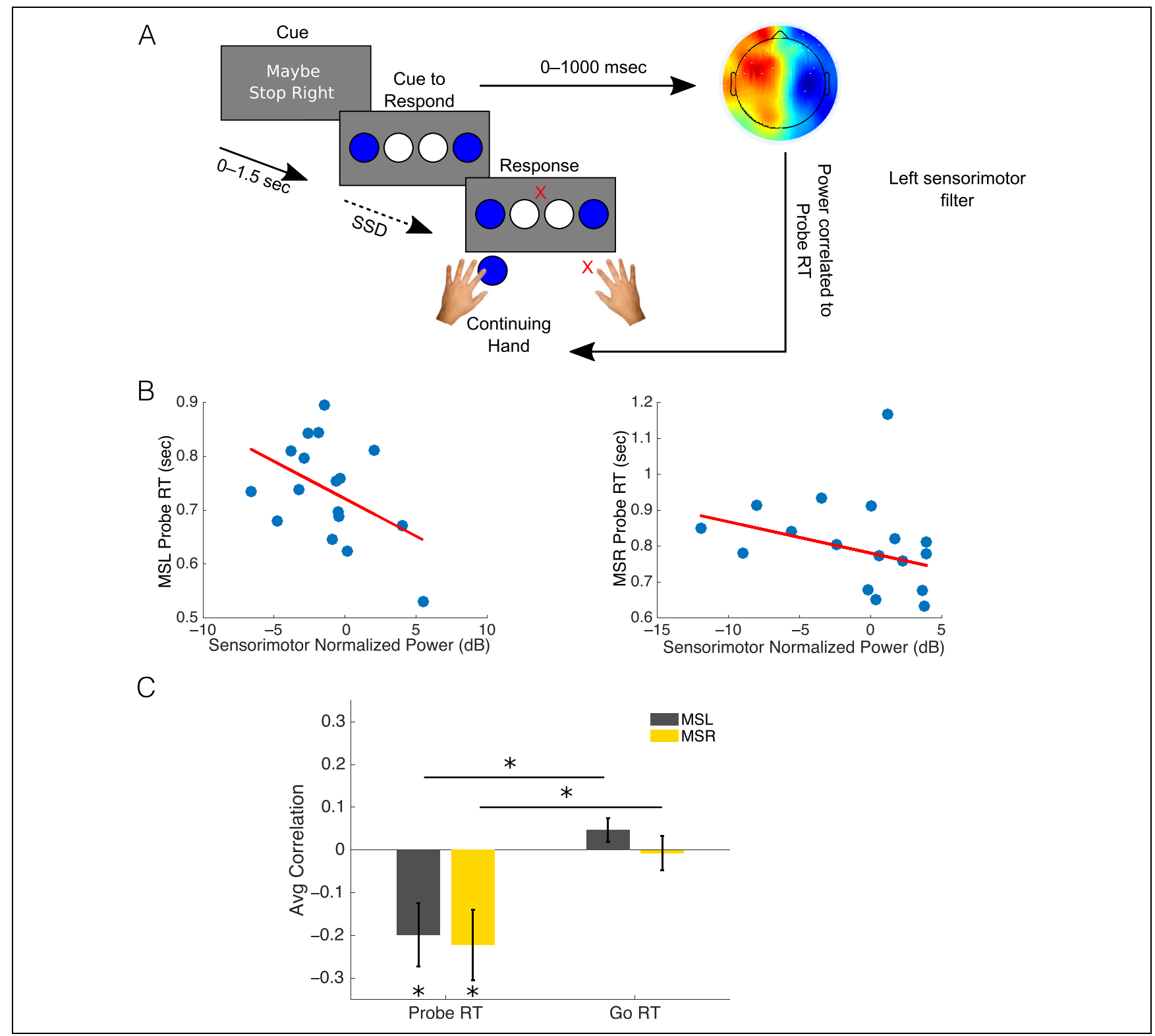

Figure 4. When preparing to stop, increased beta power relates to the behavioral selectivity of stopping. (A) An illustration of the mean power estimated for 0-1000 msec in MSR trials, which is then correlated to the probe RT, that is, the RT of the continuing hand, which in this case is the left hand. (B) The correlation between the beta power and the probe RT in both the MSL and MSR trials for a single participant. (C) The average correlation between beta and both the probe and go RTs for all participants $\left({ }^{*} p<.05\right.$; error bars represent $\left.S E M\right)$. 
was higher, the speed of continuing with the other hand was faster (i.e., stopping was more selective). This relationship was specific to the probe trials as there was no reliable correlation between beta power in the proactive period and RT on go trials without a probe $(t<2$ for MSR and MSL trials). Moreover, across participants, the correlation between proactive beta and probe alternative RT was significantly stronger than the correlation between proactive beta and go RT (MSR go vs. MSR probe, $t=$
$2.3751, p=.03 ;$ MSL go vs. MSL probe, $t=3.6276$, $p=.002$, paired $t$ test; Figure 4 ).

\section{Preparing to Stop One Hand Desynchronizes Ipsilateral Cortex in the Beta Band}

The foregoing shows that preparing to stop the hand is associated with beta power in the contralateral cortex. However, this does not tell us how people achieve this.

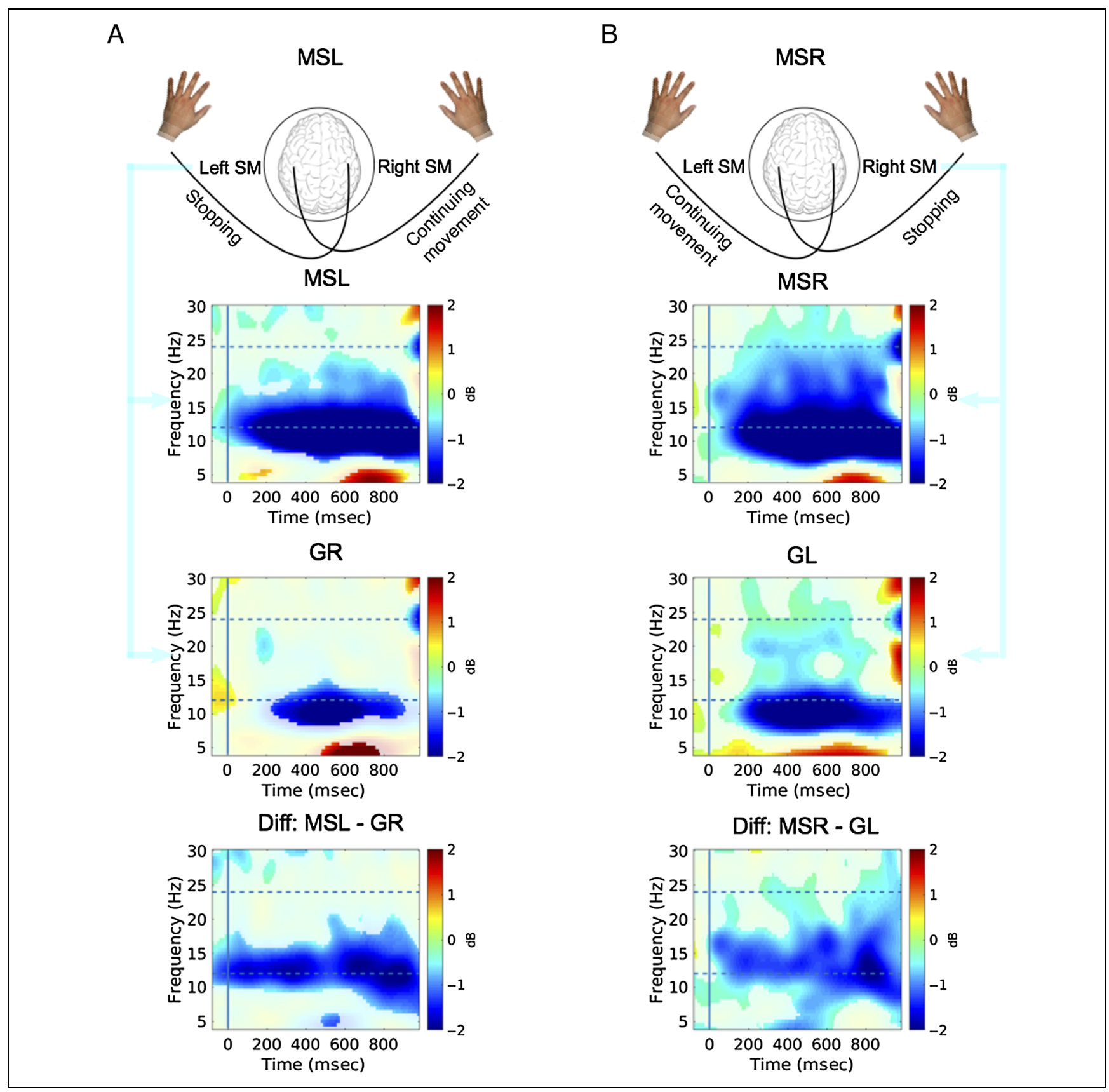

Figure 5. Preparing to stop one hand produces beta power reduction in the ipsilateral hand (for preparing to move). (A) A schematic of responses during a MSR trial and the group ERSPs for the right spatial filter during the MSR, GL trials, and their difference. (B) The same for the left spatial filter. The difference shows a desynchronization in beta, which is seen only during maybe the stop trials and not the go trials (unmasked area in plot corresponds to $p<.05$, corrected for multiple comparisons using FDR). 
One possibility is that, in this bimanual task, participants prepare the hand that needs to continue (ipsilateral to the cue to stop), and this somehow influences the contralateral side (via a putative interhemispheric interaction). Our first approach to testing this interrelation of hemispheres was to extract the power from the ipsilateral hand in the same frequency band as the contralateral. For example, if the cue was MSR, and for a participant, the right sensorimotor filter was at $15 \mathrm{~Hz}$, then we extracted $15 \mathrm{~Hz}$ power from the left sensorimotor filter. For every participant, we computed an $r$ value for the two time series (0-1000 msec) for all trials and then averaged them to get an estimate of correlation between the two hemispheres for a single subject. We then averaged this correlation across all participants. Although we anticipated a negative correlation between ipsilateral and contralateral sides, the correlations were very weak and, if anything, positive (MSR trials: $r=.02, p=.22$; MSL trials: $r=$ $.03, p<.05)$. Yet it is possible that there is an interaction between hemispheres but not necessarily in the same frequency band. We thus took a cruder approach by analyzing the data using group-level event-related spectral perturbations (ERSPs), which allows a visualization of a broader range of frequencies (here $4-30 \mathrm{~Hz}$ ). To statistically estimate significant regions in the ERSPs, we compared against zero the power for the trials at all time-frequency points in the ERSP map using unpaired $t$ tests. We then corrected for multiple comparisons using false discovery rate (FDR). All the nonsignificant regions of the ERSP map were then masked for display. As Figure 5A shows, when the cue was MSR, we plotted the ERSP from the ipsilateral (i.e., right hemisphere) filter, which corresponds to the hand that will need to definitely move/continue. At the group level, there were significant beneath-baseline power reductions (i.e., desynchronizations) in the mu and also beta bands $(p<.05$, FDR-corrected). Notably, for another condition in our design, that is, the cue GL (where participants knew for sure they would move the same left hand), the significant reduction was mostly in mu, and the difference between MSR and GL was mostly in beta. Thus, these results suggest that preparing to stop the right hand might involve preparing to move the left hand (with a desynchronization also in the beta band [12-17 Hz] rather than in the mu band for preparing to go with that hand). Below, we discuss this apparently common effect of beta synchronization (contralateral) and desynchronization (ipsilateral) in terms of a possible interaction of hemispheres. Note that the analysis of the other side for MSL was similar (Figure 5B).

\section{DISCUSSION}

We analyzed 64-channel EEG from the time period where participants prepared to stop the left or right hand. We adopted GED for performing a guided source separation analysis that created a spatial filter for each of preparing to stop the left and right hands for each participant in the beta band and based on data in the time window of interest. We show that when preparing to stop one hand there is increased beta band power in the contralateral cortex in a time window stretching from the end of the cue for about $1 \mathrm{sec}$. We verified this result with crossvalidation, using some data to derive the filters in each participant and left out data to test the hypothesis. Our next analysis was to test how this increased beta when preparing to stop is related to behavior. We show that trials with greater beta in the proactive period are ones in which the participant can subsequently stop more selectively. Finally, we tested one idea about how the participant prepares to stop, which is that he or she imagines some aspect of the other hand. We show that when preparing to stop the right hand say, there is a desynchronization in the other hemisphere that is also in the beta band.

Our first main finding was that preparing to stop was related to increased power in the beta band in contralateral cortex. We suppose that this corresponds to primary motor and premotor cortex and possibly sensory cortex. Several single-pulse TMS studies over M1 showed reduced motor-evoked potentials in the hand that was preparing to stop (Majid et al., 2013; Cai et al., 2011; Claffey et al., 2010), and an fMRI study showed increased BOLD activation in premotor cortex in the preparatory period (Majid et al., 2013). In that latter study, there was also preparatory period activation in striatum and pallidum, and moreover, people who were gene positive for Huntington's disease and had 20\% reductions of striatal volume could not stop nearly as selectively as controls, nor did they show the reduction in preparatory motorevoked potentials. Thus, taken together, the hereobserved increase of sensorimotor beta could potentially relate to premotor-cortex-basal-ganglia brain circuitry, perhaps even specific to the so-called indirect pathway of the basal ganglia.

Our second main finding was that the level of beta when preparing to stop was related to the selectivity of subsequent stopping-that is, it was functionally relevant. Notably, it did not relate to the subsequent speed of going. The way this task is set up, on trials with a probe (i.e., stop signal), the participant has to stop one hand while continuing quickly with the other. As the probe is noninformative (a red X in the center), this "forces" most participants, most of the time at least, to load the stopping rule at the start of the trial, and we have argued (Cai et al., 2011) to implement this as a proactive inhibitory influence over the motor system even before action ensues. How this putative "proactive inhibitory set" might be implemented is unclear. Based on the abovementioned findings from fMRI and in persons with Huntington's disease, one might suggest the indirect pathway of the BG, putatively targeting a subset of premotor or motor cortical neurons and somehow giving arise here, to the increased beta band power. We note 
that, although the general function of sensorimotor beta remains unclear and it may take different forms for different tasks and different periods of tasks, several studies have linked increased beta band power in sensorimotor areas to movement retardation and slower movements (Khanna \& Carmena, 2017; Pogosyan, Gaynor, Eusebio, \& Brown, 2009). It has also been related to GABA levels (Gaetz, Edgar, Wang, \& Roberts, 2011) and, in a task period that typically elicits increased beta, to protracted reduction in motor-evoked potentials (Chen, Yaseen, Cohen, \& Hallett, 1998). Furthermore, in Parkinson's disease, where akinesia is the main manifestation of the pathology, there is increased beta band coherence in the motor areas and BG (Brown, 2003, 2007). For a more general review linking beta power to an "akinetic" state, see Engel and Fries (2010). We note, however, that such results are not about beta in a preparing-to-stop scenario so much as in postmovement periods or induced artificially. There are open questions about how beta in these different scenarios might relate.

Whereas our results clearly reveal an EEG signature for preparing to stop, it is unclear how people accomplish this. It is not obvious, psychologically, what people can do with an instruction to prepare to stop. One possibility is they can somehow take this instruction and implement it as a proactive inhibitory set, involving premotor, BG, and primary motor areas, as described above. Another possibility is that they imagine moving a different effector, perhaps in the other hemisphere, and through interhemispheric interactions, this creates the suppressed state. Indeed, interhemispheric inhibition has been observed for unilateral movements (Hinder et al., 2018; Giovannelli et al., 2009) and even for imagined movement (Liang, Funase, Takahashi, Matsukawa, \& Kasai, 2014). Actually, our task explicitly required people to prepare (imagine) a bimanual movement on each trial (although they did not yet know which exact fingers would be needed). If preparing to stop the right hand, say, is related to imagining movement with the other hand, then there should be relevant changes in the ipsilateral cortex. Indeed, we observed mu and beta band desynchronizations in the ipsilateral cortex, typical of movement preparation, but notably the beta band reduction was stronger when preparing to stop compared with a pure preparing to go condition. It is interesting that this power reduction also occurred within the beta band while there was an increase of beta band power in the contralateral area. We warrant, however, that this could be an overinterpretation of the data: The relatively increased beta reduction in the ipsilateral side (for the hand getting ready to move in the stop context) could reflect any number of processes such as increased effort/fatigue/load. Further study, perhaps using causal methods, could show that this is a real push-pull relationship. This could even have clinical relevance, as one kind of approach in behavioral therapy is the so-called "competing response"-wherein performing one movement is used to counteract "suppress" the urge to do another (Himle, Woods, Piacentini, \& Walkup, 2006).

A limitation of our study is that it is unclear if the beta increase when preparing to stop is specific to this particular bimanual task or could apply more generally. Scalp EEG and electrocorticography studies of the simple stop signal task have not generally revealed beta increases in a proactive period (Wagner, Wessel, Ghahremani, \& Aron, 2018; Swann, Tandon, Pieters, \& Aron, 2013; Wessel, Conner, Aron, \& Tandon, 2013; Swann et al., 2009, 2012) nor have local field potential studies from the BG (Wessel et al., 2016; Ray et al., 2012; however, see Benis et al., 2014). Another limitation, as mentioned above, is that we cannot affirm whether the increased beta power relates to a push-pull relationship with the other hemisphere (where one can imagine movement) or something else.

Here, we focused on proactive selective suppression; however, the task also has a reactive suppression componentthat is, when participants outright stop to the stop signal. Such reactive stopping in the basic stop signal paradigm also elicits increases in beta band power, although with a frontal rather than sensorimotor topography in scalp EEG (Wagner et al., 2018). Furthermore, reactive stopping in the basic stop signal paradigm apparently has global effects, whereas the current kind of reactive stopping has selective effects (Majid, Cai, George, Verbruggen, \& Aron, 2012). These could potentially relate to different frequencies of beta (Jenkinson \& Brown, 2011). Comparing these different putative types of reactive stopping is an interesting topic for future investigation but requires having the two types of task data in each participant.

Overall, our results clearly show that preparing to stop is associated with increased beta band power in contralateral cortex and that the level of this relates to the selectivity of the subsequent stopping and not to going per se. This scalp EEG marker of proactive selective suppression (increased sensorimotor beta) could be useful for future research on selective response suppression using existing tasks (e.g., Raud \& Huster, 2017; Cowie et al., 2016; Lavallee et al., 2014; Coxon et al., 2007) and more generally for research on how people prevent provocations. It is also possible that this proactive increase in sensorimotor beta power could reflect BG indirect pathway recruitment, something that is perhaps testable in simultaneous EEG/fMRI and psychopharmacological studies. The study also highlights the substantial merits of the guided source separation procedure, in this case GED, as applied to scalp EEG. Specifically, we show here that, with priors about likely spatial topography, frequency band, and time window of interest, the method allows us to hone in on the most informative information and to test brain/behavior relationships and reveal the temporal dynamics in the motor system.

\section{Acknowledgments}

This study was supported by NIH DA026452 and the James S. McDonnell Foundation 220020375. 
Reprint requests should be sent to Adam R. Aron, Department of Psychology, University of California, San Diego, La Jolla, CA 92093, or via e-mail: adamaron@ucsd.edu.

\section{Note}

1. Data Sharing: We provide access to the EEG and behavioral data along with detailed instructions and scripts to generate the results shown in the paper: https://osf.io/zy $7 \mathrm{hn} /$.

\section{REFERENCES}

Aron, A. R., \& Verbruggen, F. (2008). Stop the presses: Dissociating a selective from a global mechanism for stopping. Psychological Science, 19, 1146-1153.

Benis, D., David, O., Lachaux, J.-P., Seigneuret, E., Krack, P., Fraix, V., et al. (2014). Subthalamic nucleus activity dissociates proactive and reactive inhibition in patients with Parkinson's disease. Neuroimage, 91, 273-281.

Blankertz, B., Tomioka, R., Lemm, S., Kawanabe, M., \& Müller, K.-R. (2008). Optimizing spatial filters for robust EEG single-trial analysis. IEEE Signal Processing Magazine, 25, 41-56.

Brainard, D. H. (1997). The Psychophysics Toolbox. Spatial Vision, 10, 433-436.

Brown, P. (2003). Oscillatory nature of human basal ganglia activity: Relationship to the pathophysiology of Parkinson's disease. Movement Disorders, 18, 357-363.

Brown, P. (2007). Abnormal oscillatory synchronisation in the motor system leads to impaired movement. Current Opinion in Neurobiology, 17, 656-664.

Cai, W., Oldenkamp, C. L., \& Aron, A. R. (2011). A proactive mechanism for selective suppression of response tendencies. Journal of Neuroscience, 31, 5965-5969.

Chen, R., Yaseen, Z., Cohen, L. G., \& Hallett, M. (1998). Time course of corticospinal excitability in reaction time and self-paced movements. Annals of Neurology, 44, $317-325$.

Claffey, M. P., Sheldon, S., Stinear, C. M., Verbruggen, F., \& Aron, A. R. (2010). Having a goal to stop action is associated with advance control of specific motor representations. Neuropsychologia, 48, 541-548.

Cohen, M. X. (2017). Comparison of linear spatial filters for identifying oscillatory activity in multichannel data. Journal of Neuroscience Methods, 278, 1-12.

Cowie, M. J., MacDonald, H. J., Cirillo, J., \& Byblow, W. D. (2016). Proactive modulation of long-interval intracortical inhibition during response inhibition. Journal of Neurophysiology, 116, 859-867.

Coxon, J. P., Stinear, C. M., \& Byblow, W. D. (2007). Selective inhibition of movement. Journal of Neurophysiology, 97, 2480-2489.

Duque, J., Greenhouse, I., Labruna, L., \& Ivry, R. B. (2017). Physiological markers of motor inhibition during human behavior. Trends in Neurosciences, 40, 219-236.

Engel, A. K., \& Fries, P. (2010). Beta-band oscillationssignalling the status quo? Current Opinion in Neurobiology, 20, 156-165.

Gaetz, W., Edgar, J. C., Wang, D. J., \& Roberts, T. P. L. (2011). Relating MEG measured motor cortical oscillations to resting gamma-aminobutyric acid (GABA) concentration.

Neuroimage, 55, 616-621.

Giovannelli, F., Borgheresi, A., Balestrieri, F., Zaccara, G., Viggiano, M. P., Cincotta, M., et al. (2009). Modulation of interhemispheric inhibition by volitional motor activity: An ipsilateral silent period study. Journal of Physiology, 587, 5393-5410
Haufe, S., Meinecke, F., Görgen, K., Dähne, S., Haynes, J.-D., Blankertz, B., et al. (2014). On the interpretation of weight vectors of linear models in multivariate neuroimaging. Neuroimage, 87, 96-110.

Himle, M. B., Woods, D. W., Piacentini, J. C., \& Walkup, J. T. (2006). Brief review of habit reversal training for Tourette syndrome. Journal of Child Neurology, 21, 719-725.

Hinder, M. R., Puri, R., Kemp, S., Waitzer, S., Reissig, P., Stöckel, T., et al. (2018). Distinct modulation of interhemispheric inhibitory mechanisms during movement preparation reveals the influence of cognition on action control. Cortex, 99, 13-29.

Jenkinson, N., \& Brown, P. (2011). New insights into the relationship between dopamine, beta oscillations and motor function. Trends in Neurosciences, 34, 611-618.

Khanna, P., \& Carmena, J. M. (2017). Beta band oscillations in motor cortex reflect neural population signals that delay movement onset. elife, 6, e24573.

Kilavik, B. E., Zaepffel, M., Brovelli, A., MacKay, W. A., \& Riehle, A. (2013). The ups and downs of beta oscillations in sensorimotor cortex. Experimental Neurology, 245, $15-26$.

Lavallee, C. F., Meemken, M. T., Herrmann, C. S., \& Huster, R. J. (2014). When holding your horses meets the deer in the headlights: Time-frequency characteristics of global and selective stopping under conditions of proactive and reactive control. Frontiers in Human Neuroscience, 8,994 .

Liang, N., Funase, K., Takahashi, M., Matsukawa, K., \& Kasai, T. (2014). Unilateral imagined movement increases interhemispheric inhibition from the contralateral to ipsilateral motor cortex. Experimental Brain Research, 232, 1823-1832.

Logan, G. D., \& Cowan, W. B. (1984). On the ability to inhibit thought and action: A theory of an act of control. Psychological Review, 91, 295-327.

Majid, D. S. A., Cai, W., Corey-Bloom, J., \& Aron, A. R. (2013). Proactive selective response suppression is implemented via the basal ganglia. Journal of Neuroscience, 33, 13259-13269.

Majid, D. S. A., Cai, W., George, J. S., Verbruggen, F., \& Aron, A. R. (2012). Transcranial magnetic stimulation reveals dissociable mechanisms for global versus selective corticomotor suppression underlying the stopping of action. Cerebral Cortex, 22, 363-371.

Makeig, S., Bell, A. J., Jung, T.-P., \& Sejnowski, T. J. (1996). Independent component analysis of electroencephalographic data. In D. Touretzky, M. Mozer, \& M. Hasselmo (Eds.), Advances in neural information processing systems (Vol. 8, pp. 145-151). Cambridge, MA: MIT Press.

Parra, L., \& Sajda, P. (2004). Blind source separation via generalized eigenvalue decomposition. Journal of Machine Learning Research, 4, 1261-1269.

Pfurtscheller, G., \& Solis-Escalante, T. (2009). Could the beta rebound in the EEG be suitable to realize a "brain switch"? Clinical Neurophysiology, 120, 24-29.

Pogosyan, A., Gaynor, L. D., Eusebio, A., \& Brown, P. (2009). Boosting cortical activity at Beta-band frequencies slows movement in humans. Current Biology, 19, 1637-1641.

Raud, L., \& Huster, R. J. (2017). The temporal dynamics of response inhibition and their modulation by cognitive control. Brain Topography, 30, 486-501.

Ray, N. J., Brittain, J.-S., Holland, P., Joundi, R. A., Stein, J. F., Aziz, T. Z., et al. (2012). The role of the subthalamic nucleus in response inhibition: Evidence from local field potential recordings in the human subthalamic nucleus. Neuroimage, 60, 271-278. 
Smittenaar, P., Guitart-Masip, M., Lutti, A., \& Dolan, R. J. (2013). Preparing for selective inhibition within frontostriatal loops. Journal of Neuroscience, 33, 18087-18097.

Swann, N. C., Cai, W., Conner, C. R., Pieters, T. A., Claffey, M. P., George, J. S., et al. (2012). Roles for the pre-supplementary motor area and the right inferior frontal gyrus in stopping action: Electrophysiological responses and functional and structural connectivity. Neuroimage, 59, 2860-2870.

Swann, N. C., Tandon, N., Canolty, R., Ellmore, T. M., McEvoy, L. K., Dreyer, S., et al. (2009). Intracranial EEG reveals a timeand frequency-specific role for the right inferior frontal gyrus and primary motor cortex in stopping initiated responses. Journal of Neuroscience, 29, 12675-12685.

Swann, N. C., Tandon, N., Pieters, T. A., \& Aron, A. R. (2013). Intracranial electroencephalography reveals different temporal profiles for dorsal- and ventro-lateral prefrontal cortex in preparing to stop action. Cerebral Cortex, 23, 2479-2488.
Wagner, J., Wessel, J. R., Ghahremani, A., \& Aron, A. R. (2018). Establishing a right frontal beta signature for stopping action in scalp EEG: Implications for testing inhibitory control in other task contexts. Journal of Cognitive Neuroscience, 30 , 107-118.

Wessel, J. R., Conner, C. R., Aron, A. R., \& Tandon, N. (2013). Chronometric electrical stimulation of right inferior frontal cortex increases motor braking. Journal of Neuroscience, 33, 19611-19619.

Wessel, J. R., Ghahremani, A., Udupa, K., Saha, U., Kalia, S. K., Hodaie, M., et al. (2016). Stop-related subthalamic beta activity indexes global motor suppression in Parkinson's disease. Movement Disorders, 31, 1846-1853.

Xu, J., Westrick, Z., \& Ivry, R. B. (2015). Selective inhibition of a multicomponent response can be achieved without cost. Journal of Neurophysiology, 113, 455-465. 